\title{
LOCALLY $B^{*}$-EQUIVALENT ALGEBRAS. II( ${ }^{1}$ )
}

\author{
BY
}

\author{
BRUCE A. BARNES
}

ABSTRACT. Let $A$ be a locally $B^{*}$-equivalent Banach *-algebra. Then $A$ possesses a unique norm $|\cdot|$ with the property that $\left|a^{*} a\right|=|a|^{2}$ for all $a \epsilon A$. Let $B$ be the $B^{*}$-algebra which is the completion of $A$ in the norm $|\cdot|$. In this paper it is shown that there exists a closed $B^{*}$-equivalent ${ }^{*}$-ideal of $A$ which contains the maximal GCR ideal of $B$. In particular, when $B$ is a GCR algebra, then $A=B$.

1. Introduction. Let $A$ be a Banach ${ }^{*}$-algebra. $A$ is $B^{*}$-equivalent if $A$ is *-isomorphic to some $B^{*}$-algebra $B$. Then by $[6$, Theorem (4.1.20)] and the Closed Graph Theorem, the *-isomorphism between $A$ and $B$ is a homeomorphism. Thus $A$ and $B$ have the same algebraic and topological structure (but not necessarily the same geometric structure). We call $A$ locally $B^{*}$-equivalent if for every selfadjoint element $a \in A$, the closed *-subalgebra of $A$ generated by $a$ is $B^{*}$ equivalent. Whether or not every locally $B^{*}$-equivalent Banach *-algebra is $B^{*}$ equivalent is not known. Some partial results on this question have been obtained in [2].

Now assume that $A$ is a locally $B^{*}$-equivalent Banach ${ }^{*}$-algebra. Then $A$ possesses a unique norm $|\cdot|$ with the property that $\left|a^{*} a\right|=|a|^{2}$ for all $a \in A$. Let $B$ be the $B^{*}$-algebra which is the completion of $A$ in the norm $|\cdot|$. In this paper, which is a sequel to [2], we prove that there exists a closed $B^{*}$-equivalent *-ideal of $A$ which contains the maximal GCR ideal of $B$ (Theorem 2). Thus if $B$ is a GCR algebra, then $A=B$.

$\mathrm{H}$. Behncke has considered some questions concerning locally $B^{*}$-equivalent algebras in [3]. However, there is almost no overlap of his paper with either this paper or [2].

2. Preliminary remarks. Throughout this paper $A$ is a locally $B^{*}$-equivalent Banach *algebra with norm $\|\cdot\|$. By [2, Proposition 2.1], there is a unique norm $|\cdot|$ on $A$ with the $B^{*}$-property (that is, $\left|a^{*} a\right|=|a|^{2}$ for all $a \in A$ ). Throughout this paper $B$ is the completion of $A$ with respect to the norm $|\cdot|$. Then $A$ is $B^{*}$-equivalent if and only if $A=B$.

By $[6$, Corollary (4.1.16) $]$, there exists a constant $M>0$ such that $|a| \leq$ $M\|a\|$ for all $a \in A$. Since $A$ is locally $B^{*}$-equivalent, the norms $\|\cdot\|$ and $|\cdot|$

Received by the editors March 1, 1972.

AMS (MOS) subject classifications (1970). Primary 46L05, 46K99, 46H 20.

(1) This research was partially supported by National Science Foundation Grant GP-28250. 
are equivalent on the closed *-subalgebra of $A$ generated by a selfadjoint element of $A$. Therefore, if $a=a^{*} \in A$, there exists $K>0$ such that $\left\|a^{n}\right\| \leq K\left|a^{n}\right|$ for all $n \geq 1$. Then

$$
\left\|a^{n}\right\|^{1 / n} \leq K^{1 / n}\left|a^{n}\right|^{1 / n}=K^{1 / n}|a| .
$$

It follows that the spectral radius of a selfadjoint element $a$ is $|a|$.

The norms $\|\cdot\|$ and $|\cdot|$ are equivalent on every maximal commutative *-subalgebra of $A$ by [2, Proposition 2.2]. This implies a fact that we use repeatedly: if $\left\{a_{n}\right\}$ is a sequence of selfadjoint elements of $A$ and $a_{n} a_{m}=0$ whenever $n \neq m$, then there exists $K>0$ such that $\left\|a_{n}\right\| \leq K\left|a_{n}\right|$ for all $n \geq 1$ (proof-the sequence $\left\{a_{n}\right\}$ is contained in some maximal commutative ${ }^{*}$-subalgebra of $A$ ).

3. Preliminary results. In this section we prove some basic lemmas. If $I$ is a $|\cdot|$-closed ideal of $A$, we let $|\cdot|_{q}$ denote the usual quotient norm on $A / I$,

$$
|a+I|_{q}=\inf \{|a-b| \mid b \in I\}
$$

Lemma 1. Let I be a closed ideal of B. Then

(1) $A \cap I$ is $|\cdot|$-dense in $I$, and

(2) if $\|\cdot\|$ and $|\cdot|$ are equivalent on $A \cap I$, then $I \subset A$.

Proof. Since $I$ is a closed ideal in the $B^{*}$-algebra $B, I$ is a ${ }^{*}$-ideal (see [6, Theorem (4.9.2)]). Then $A \cap I$ is a $|\cdot|$-closed (and therefore also $\|\cdot\|$ closed) *-ideal of $A$. Let $J$ be the $|\cdot|$-closure of $A \cap I$ in $B$. Then $A \cap J=$ $A \cap I$. The quotient algebra $A / A \cap I$ is naturally embedded in the quotient algebras $B / I$ and $B / J$ by the maps $a+A \cap I \rightarrow a+I$ and $a+A \cap I \rightarrow a+J$, respectively. Define two norms on $A / A \cap I$ by

$$
|a+A \cap I|_{1}=|a+I|_{q} \text { and }|a+A \cap I|_{2}=|a+J|_{q}
$$

Since $|a+I|_{q}$ and $|a+J|_{q}$ are $B^{*}$-norms, then $|\cdot|_{1}$ and $|\cdot|_{2}$ are norms on $A / A \cap I$ that have the $B^{*}$-property. By [2, Proposition 2.3], $A / A \cap I$ is locally $B^{*}$-equivalent. Then by [2, Proposition 2.1], $|a+A \cap I|_{1}=|a+A \cap I|_{2}$ for all $a \in A$. It follows that $|a+I|_{q}=|a+J|_{q}$ for all $a \in A$. Now assume $t \in I$, and choose a sequence $\left\{t_{n}\right\}$ in $A$ such that $\left|t_{n}-t\right| \rightarrow 0$. Then $\left|\left(t_{n}-t\right)+I\right|_{q} \rightarrow 0$, and since $t \in I$, it follows that $\left|t_{n}+I\right|_{q} \rightarrow 0$. Therefore $\left|t_{n}+J\right|_{q} \rightarrow 0$. This means that there exists $\left\{s_{n}\right\} \subset J$ such that $\left|t_{n}-s_{n}\right| \rightarrow 0$. Then $\left|t-s_{n}\right| \rightarrow 0$, so that $t \in J$. This proves (1).

Now assume that $\|\cdot\|$ and $|\cdot|$ are equivalent on $A \cap I$. If $t \in I$, by (1) we can choose $\left\{t_{n}\right\} \subset A \cap I$ such that $\left|t_{n}-t\right| \rightarrow 0$. Then the sequence $\left\{t_{n}\right\}$ is $|\cdot|-$ Cauchy, and therefore $\|\cdot\|$-Cauchy. Therefore there exists $s \in A$ such that $\left\|t_{n}-s\right\| \rightarrow 0$. Then $\left|t_{n}-s\right| \rightarrow 0$ so that $s=t$. 
If $a=a^{*} \in A$, let $C(a)$ be the closed *-subalgebra of $A$ generated by $a$. Let $\Phi_{C(a)}$ be the carrier space of $C(a)$, and if $b \in C(a)$, let $\hat{b}$ denote the Gelfand transform of $b$. Since $A$ is locally $B^{*}$-equivalent, $b \rightarrow \hat{b}$ is a bicontinuous *-isomorphism of $C(a)$ onto the algebra of all complex continuous functions that vanish at infinity on $\Phi_{C(a)}$. Now let $D$ be a *-subalgetra of $A$. By [2, Lemma 2.5], if there exists $K>0$ such that $K|a| \geq\|a\|$ for all $a=a^{*} \in D$, then the $\|\cdot\|$ closure of $D$ is $B^{*}$-equivalent. We use these results in the proof of the next lemma.

Lemma 2. Let I be $a^{*}$-subalgebra of $A$ with the properties:

(1) if $t^{*}=t \in I$, then $C(t) \subset I$,

(2) if $t \in I$, then $\left(1-t^{*}\right) I(1-t) \neq\{0\}$.

Then there exists $b \in I$ such that the $\|\cdot\|$-closure of $\left(1-b^{*}\right) I(1-b)$ is $B^{*}$. equivalent.

Proof. Suppose that there is no $b \in I$ such that the $\|\cdot\|$-closure of $\left(1-b^{*}\right) I(1-b)$ is $B^{*}$-equivalent. Then the $\|\cdot\|$-closure of $I$ is not $B^{*}$-equivalent, so that there exists $b_{1}^{*}=b_{1} \in I$ such that $\left|b_{1}\right|<1 / 2\left\|b_{1}\right\|$. Choose $g_{1}^{*}=g_{1} \in C\left(b_{1}\right)$ such that $\hat{g}_{1}$ has compact support in $\Phi_{C\left(b_{1}\right)}$ and $\left|g_{1}\right|<1 / 2\left\|g_{1}\right\|$. Then choose $k_{1}^{*}=k_{1} \in C\left(b_{1}\right)$ such that $\hat{k}_{1} \equiv 1$ on the support of $\hat{g}_{1}$. Note that $g_{1}\left(1-k_{1}\right)$ $\left(1-k_{1}\right) g_{1}=0$. Let $I_{1}-\left(1-k_{1}\right) I\left(1-k_{1}\right)$. By assumption the $\|\cdot\|$-closure of $I_{1}$ is not $B^{*}$-equivalent. Then choose $b_{2}^{*}=b_{2} \in I_{1}$ such that $\left|b_{2}\right|<1 / 4\left\|b_{2}\right\|$. Choose as before $g_{2}^{*}=g_{2}$ and $k_{2}^{*}=k_{2}$ in $C\left(b_{2}\right)$ such that $\left|g_{2}\right|<1 / 4\left\|g_{2}\right\|$ and $g_{2}\left(1-k_{2}\right)=$ $\left(1-k_{2}\right) g_{2}=0$. Since $g_{1} b_{2}=b_{2} g_{1}=0$, then $g_{1} k_{2}=k_{2} g_{1}=0$ and $g_{1} g_{2}=g_{2} g_{1}=0$. Let $I_{2}=\left(1-k_{2}\right)\left(1-k_{1}\right) I\left(1-k_{1}\right)\left(1-k_{2}\right)$. If $u=\left(1-k_{2}\right)\left(1-k_{1}\right) v\left(1-k_{1}\right)\left(1-k_{2}\right)$ for some $v \in I$, then $g_{1} u=u g_{1}=0$ and $g_{2} u=u g_{2}=0$. As before choose $g_{3}=g_{3}^{*}$ and $k_{3}=k_{3}^{*}$ in $I$ such that $\left|g_{3}\right|<(1 / 2)^{3}\left\|g_{3}\right\|,\left(1-k_{3}\right) g_{3}=g_{3}\left(1-k_{3}\right)=0$, and $g_{i} g_{j}=0$ if $i \neq j$. Continuing in this fashion we can construct a sequence of selfadjoint elements $\left\{g_{k}\right\} \subset I$ such that $g_{i} g_{j}=0$ whenever $i \neq j$ and $\left|g_{k}\right|<(1 / 2)^{k}\left\|g_{k}\right\|$. This contradicts the fact that $A$ is locally $B^{*}$-equivalent.

Lemma 3. Let $\left\{I_{\lambda}\right\}, \lambda \in \Lambda$, be a collection of closed ${ }^{*}$-ideals of $A$, and let $I=\bigcup_{\lambda \in \mathbf{\Lambda}} I_{\lambda^{*}}$. If $I_{\boldsymbol{\lambda}}$ is $B^{*}$-equivalent for each $\lambda \in \Lambda$, and $I$ is a ${ }^{*}$-subalgebra of $A$, then the $\|\cdot\|$-closure of $I$ is $B^{*}$-equivalent.

Proof. Assume that there exists an element $t \in I$ such that $\left(1-t^{*}\right) I(1-t)=$ \{0\}. Then for any $a \in I,\left(1-t^{*}\right) a^{*} a(1-t)=0$, so that $a(1-t)=0$. There exists $\mu \in \Lambda$ such that $t \in I_{\mu}$. Then $a=a t \in I_{\mu}$. Then $I=I_{\mu}$, which proves the lemma in this case.

Now assume that $\left(1-t^{*}\right) I(1-t) \neq\{0\}$ whenever $t \in I$. By Lemma 2 there exists $b \in I$ such that the $\|\cdot\|$-closure of $\left(1-b^{*}\right) I(1-b)$ is $B^{*}$-equivalent. Assume that $\left\{b_{n}\right\}$ is a $|\cdot|$-Cauchy sequence in the $\|\cdot\|$-closure of $I$. For each $n$, 


$$
b_{n}=\left[\left(1-b^{*}\right) b_{n}(1-b)\right]+\left[\left(1-b^{*}\right) b_{n} b+b^{*} b_{n}\right] .
$$

There exists $\mu \in \Lambda$ such that $b \in I_{\mu}$. Then $\left\{\left(1-b^{*}\right) b_{n} b+b^{*} b_{n}\right\}$ is a $|\cdot|$-Cauchy sequence in $I_{\mu}$, and $\left\{\left(1-b^{*}\right) b_{n}(1-b)\right\}$ is a $|\cdot|$-Cauchy sequence in the $\|\cdot\|$ closure of $\left(1-b^{*}\right) I(1-b)$. Since both these $|\cdot|$-Cauchy sequences converge to some element of $A$, then $\left\{b_{n}\right\}$ converges to some element of $A$. It follows that the $\|\cdot\|$-closure of $I$ is $B^{*}$-equivalent.

4. Locally $B^{*}$-equivalent algebras of vector valued functions. Let $\Omega$ be a compact Hausdorff space and denote by $C_{R}(\Omega)$ the algebra of all continuous real-valued functions on $\Omega$. For each $\omega \in \Omega$, let $B_{\omega}$ be a $B^{*}$-algebra with norm $|\cdot|_{\omega}$. Define $C\left(\Omega, B_{\omega}\right)$ to be the algebra of all functions $f$ defined on $\Omega$ such that $f(\omega) \in B_{\omega}$ for all $\omega \in \Omega$ and such that the function $\omega \rightarrow|f(\omega)|_{\omega}$ is in $C_{R}(\Omega)$. The algebra $C\left(\Omega, B_{\omega}\right)$ is a $B^{*}$-algebra in the norm

$$
|f|=\sup _{\omega \in \Omega}|f(\omega)|_{\omega} .
$$

Throughout this section we assume that $B$ is a closed $*_{\text {-subalgebra of } C\left(\Omega, B_{\omega}\right)}$ which is closed under multiplication by functions in $C_{R}(\Omega)$.

Theorem 1. Assume that $A$ is a dense *-subalgebra of $B$ sucb that $A$ is a locally $B^{*}$-equivalent Banach algebra. Furthermore, assume that if $\omega_{1}, \cdots, \omega_{n}$ are any $n$ distinct points in $\Omega$ and $b_{1}, \cdots, b_{n}$ are such that $b_{k} \in B_{\omega_{k}}, 1 \leq k \leq n$, then there exists $g \in A$ such that $g\left(\omega_{k}\right)=b_{k}, 1 \leq k \leq n$.

Then $A=B$.

We proceed with the proof of Theorem 1 by establishing several lemmas. If $V$ is a subset of $\Omega$, let $J_{V}=\{f \in B \mid f(\omega)=0$ whenever $\omega \notin V\}$. We say that $\|\cdot\|$ and $|\cdot|$ are equivalent on $A$ at $\omega \in \Omega$ if there exists an open neighborhood $V$ of $\omega$ such that $\|\cdot\|$ and $|\cdot|$ are equivalent on the ideal $A \cap J_{V}$.

Lemma 4. The norms $\|\cdot\|$ and $|\cdot|$ are equivalent on $A$ at all but at most a finite number of points of $\Omega$.

Proof. Suppose that there is an infinite sequence of distinct points $\left\{\omega_{n}\right\} \subset \Omega$ such that $\|\cdot\|$ and $|\cdot|$ are not equivalent on $A$ at $\omega_{n}$ for all $n \geq 1$. By choosing a suitable subsequence of $\left\{\omega_{n}\right\}$ if necessary, we may assume that there exists a disjoint sequence of open subsets of $\Omega,\left\{U_{n}\right\}$, such that $\omega_{n} \in U_{n}$ for $n \geq 1$. Then for each $n \geq 1$ there exists $g_{n}^{*}=g_{n} \in J_{U_{n}} \cap A$ such that $n\left|g_{n}\right|<\left\|g_{n}\right\|$. Since $g_{n} g_{m}=g_{m} g_{n}=0$ when $n \neq m$, this contradicts the assumption that $A$ is locally $B^{*}$-equivalent.

If $|\cdot|$ and $\|\cdot\|$ are equivalent on $A$ at every point in $\Omega$, let $I=B$. In the case where there exist points in $\Omega$ at which $|\cdot|$ and $\|\cdot\|$ are not equivalent on $A$, then by Lemma 4 there must be only a finite number of such points. Let 
$\left\{\omega_{1}, \cdots, \omega_{n}\right\}$ be this collection of points. Define $I$ in this case to be the set of all $f \in B$ such that $f\left(\omega_{k}\right)=0,1 \leq k \leq n$.

Lemma 5. $I \subset A$.

Proof. For each $\omega \in \Omega$ such that $\omega \neq \omega_{k}, 1 \leq k \leq n$, there exists an open neighborhood $U$ of $\omega$ in $\Omega$ such that $|\cdot|$ and $\|\cdot\|$ are equivalent on $A \cap J_{U}$. It follows from Lemma 1 that $J_{U} \subset A$. Let $K$ be a closed subset of $\Omega$ disjoint from $\left\{\omega_{1}, \cdots, \omega_{n}\right\}$ (when $I=B$, let $K=\Omega$ ). Choose $\left\{U_{1}, \cdots, U_{m}\right\}$ a finite open cover of $K$ such that $|\cdot|$ and $\|\cdot\|$ are equivalent on $A \cap J_{U_{k}}, 1 \leq k \leq m$. Then, as noted above, $J_{U_{k}} \subset A, 1 \leq k \leq m$. Let $U_{0}=\Omega \backslash K$. Choose $f_{k} \in C_{R}(\Omega)$ such that $f_{k}(\omega)=0$ whenever $\omega \notin U_{k}, 0 \leq k \leq m$, and such that $1=\sum_{k=0}^{m} f_{k}$. If $g \in J_{K}$, then $f_{0} g=0$. Then $g=\sum_{k=0}^{m} f_{k} g=\sum_{k=1}^{m} f_{k} g$. By hypothesis $B$ is closed under multiplication by functions in $C_{R}(\Omega)$. Therefore $f_{k} g \in J_{U_{k}} \subset A$ for $1 \leq k \leq m$. (In the case where $I=B$, the proof is now complete.) There exist constants $M_{k}$ such that $\|b\| \leq M_{k}|b|$ whenever $b \in J_{U_{k}}, 1 \leq k \leq m$. Then

$$
\|g\| \leq \sum_{k=1}^{m}\left\|f_{k} g\right\| \leq \sum_{k=1}^{m} M_{k}\left|f_{k} g\right| \leq\left(\sum_{k=1}^{m} M_{k}\left\|f_{k}\right\|_{\infty}\right)|g| .
$$

Therefore $|\cdot|$ and $\|\cdot\|$ are equivalent on the closed ${ }^{*}$-ideal $J_{K} \subset A$. Let $I_{0}=$ U $J_{K}$ where the union is over all closed subsets $K$ of $\Omega$ that are disjoint from $\left\{\omega_{1}, \cdots, \omega_{n}\right\}$. By Lemma $3,|\cdot|$ and $\|\cdot\|$ are equivalent on the $\|\cdot\|$-closure of $I_{0}$. But $I_{0}$ is $|\cdot|$-dense in $I$. Therefore the same proof as given for part (2) of Lemma 1 shows that $I \subset A$.

Now we complete the proof of Theorem 1. Assume that $f \in B$. Let $\left\{\omega_{1}, \cdots, \omega_{n}\right\}$ be as above. By hypothesis there exists $g \in A$ such that $g\left(\omega_{k}\right)=f\left(\omega_{k}\right), 1 \leq k$ $\leq n$. Then $f-g \in I$. But by Lemma $5, I \subset A$, and therefore $f \in A$.

5. The main result. Assume that $B$ is a CCR (liminaire) algebra with Hausdorff structure space $\Omega$. To each point $P \in \Omega$ associate the $B^{*}$-algebra $B_{P}=B / P$. Each of the algebras $B_{P}$ is *-isomorphic to the algebra of all compact operators on some Hilbert space. By [5, Lemma 4.3] $\Omega$ is locally compact in the Jacobson topology. If $\Omega$ is not compact, then compactify it by adding a point at $\infty, P_{\infty}$, and associate with $P_{\infty}$ the algebra $B_{P_{\infty}}=\{0\}$. Each $b \in B$ determines a function $b(P)$ defined on $\Omega$ by

$$
b(P)=b+P \in B_{P} .
$$

Let $|b(P)|_{P}$ be the quotient norm of $b+P$ in $B / P(b \in B, P \in \Omega)$. Then $|b|=$ $\sup \left\{|b(P)|_{P} \mid P \in \Omega\right\}$ for all $b \in B$. Furthermore, by [5, Theorem 4.1], $P \rightarrow$ $|b(P)|_{P}$ is a continuous function on $\Omega$. If $P_{1}, \cdots, P_{n}$ are $n$ distinct points of $\Omega$, and $b_{1}, \cdots, b_{n}$ are $n$ elements of $B$, then there exists $b \in B$ such that 
$b\left(P_{k}\right)=b+P_{k}=b_{k}+P_{k}$ for $1 \leq k \leq n$. This follows from [4, Proposition 4.2] for the case $n=2$, but the result is easily extended to all positive integers $n$. Then [5, Theorem 3.3] applies to $B$, so that $B$ is closed under multiplication by functions in $C_{R}(\Omega)$.

Now assume that $A$ is a locally $B^{*}$-equivalent Banach algebra which is a dense *-subalgebra of $B$. Let $P_{1}, \cdots, P_{n}$ be $n$ distinct points of $\Omega$. Let $B^{\prime}=$ $B_{P_{1}} \oplus \cdots \oplus B_{P_{n}}$ (the direct sum of the quotient algebras $B_{P_{k}}, 1 \leq k \leq n$ ). $B^{\prime}$ is a dual $B^{*}$-algebra. Let $I=A \cap P_{1} \cap \cdots \cap P_{n}$. The map $\pi: A / I \rightarrow B^{\prime}$, defined for $a \in A$ by $\pi(a+I)=\left(a+P_{1}\right) \oplus \cdots \oplus\left(a+P_{n}\right)$, is a *-isomorphism of the locally $B^{*}$-equivalent Banach algebra $A / I$ into $B^{\prime}$. Let $b_{1}, \cdots, b_{n}$ be any $n$ elements of $B$. Then as noted above, there exists $b \in B$ such that $b\left(P_{k}\right)=b+P_{k}$ $=b_{k}+P_{k}$ for $1 \leq k \leq n$. Choose a sequence $\left\{a_{j}\right\} \subset A$ such that $\left|a_{j}-b\right| \rightarrow 0$. Then $\pi\left(a_{j}+I\right) \rightarrow\left(b_{1}+P_{1}\right) \oplus \cdots \oplus\left(b_{n}+P_{n}\right)$ in $B^{\prime}$. This proves that $A^{\prime}=$ $\pi(A / I)$ is a dense locally $B^{*}$-equivalent Banach ${ }^{*}$-subalgebra of the dual $B^{*}$. algebra $B^{\prime}$. If $t=t^{*} \in A^{\prime}$, then $t$ has the same spectrum in $A^{\prime}$ and $B^{\prime}$. Then by [1, Theorem 3.4], the spectrum of $t$ in $A^{\prime}$ is at most countable. Therefore by [2, Theorem 4.1], $A^{\prime}=B^{\prime}$. It follows that if $b_{1}, \cdots, b_{n}$ are $n$ elements of $B$, there exists $a \in A$ such that $a\left(P_{k}\right)=b_{k}+P_{k}$ for $1 \leq k \leq n$. Then $A$ satisfies all the hypotheses of Theorem 1 , so that $A=B$. We state this result as a proposition.

Proposition 6. If $B$ is a CCR algebra with Hausdorff structure space, then $A=B$.

Lemma 7. Let $K$ be a closed $B^{*}$-equivalent ${ }^{*}$-ideal of $A$. Then there exists a closed $B^{*}$-equivalent ${ }^{*}$-ideal $I$ of $A$ such that $K \subset I$ and $A / I$ bas no nonzero closed $B^{*}$-equivalent ${ }^{*}$-ideals.

Proof. Let 9 be the collection of all closed $B^{*}$-equivalent ${ }^{*}$-ideals of $A$ that contain $K$. Then 9 is nonempty, and 9 is partially ordered by inclusion. Assume that $\left\{I_{\lambda}\right\}, \lambda \in \Lambda$, is a chain in 9 . Then by Lemma 3 the $\|\cdot\|$-closure of $\bigcup_{\lambda \in \Lambda} I_{\lambda}$ is in 9 . By Zorn's Lemma 9 contains a maximal element $I$. Assume that $J$ is a closed $B^{*}$-equivalent ${ }^{*}$-ideal of $A /$ I. Let $\pi$ be the natural quotient map of $A$ onto $A / I$. By [2, Lemma 2.4], $\pi^{-1}(J)$ is a closed $B^{*}$-equivalent ${ }^{*}$-ideal of $A$ that contains $I$. Then by the maximality of $I, \pi^{-1}(J)=I$. This proves that $J$ is the zero ideal in $A / l$.

Proposition 8. If $B$ is a GCR algebra, then $A=B$.

Proof. By Lemma $7 A$ contains a closed $B^{*}$-equivalent ${ }^{*}$-ideal $I$ such that $A / I$ has no nonzero closed $B^{*}$-equivalent ${ }^{*}$-ideals. Then $I$ is a closed ${ }^{*}$-ideal of $B$, and $A / I$ is a dense locally $B^{*}$-equivalent *-subalgebra of $B / I$. By $[4$, Proposition 4.3.5], $B / I$ is a GCR algebra. Let $A_{1}$ and $B_{1}$ denote the quotient 
algebras $A / I$ and $B / I$, respectively. Assume $B_{1} \neq\{0\}$ (that is, $B \neq I$ ). Then $B_{1}$ contains a nonzero closed ${ }^{*}$-ideal $J$ which is a CCR algebra (see [4, p. 87]). Then $J_{1}$, the first nonzero ideal in the composition series described in $[5$, Theorem 6.2], is a closed *-ideal of $B_{1}$ which is a CCR algebra with Hausdorff structure space. Now $A_{1} \cap J_{1}$ is dense in $J_{1}$ by Lemma 1 . Then by Proposition 6, $J_{1} \subset A_{1}$. This contradiction implies that $B=I$. Therefore $B=A$.

Now we prove the main result.

Theorem 2. There exists a closed *-ideal I of $A$ with the properties:

(1) $I$ is $B^{*}$-equivalent.

(2) I contains the largest GCR ideal of $B$, and

(3) $A / I$ has no nonzero closed $B^{*}$-equivalent ideals.

Proof. Let $J$ be the largest GCR ideal of $B$ (see [5, Theorem 7.5]). By Lemma $1 A \cap J$ is a dense *-subalgebra of $J$. Furthermore $A \cap J$ is locally $B^{*}$ equivalent. Then by Proposition $8, J=A \cap J \subset A$. Then Lemma 7 implies the existence of a closed *-ideal $I$ of $A$ with properties (1)-(3).

\section{REFERENCES}

1. B. A. Barnes, A generalized Fredholm theory for certain maps in the regular representations of an algebra, Canad. J. Math. 20 (1968), 495-504. MR 38 \#534.

2. - L Locally $B^{*}$-equivalent algebras, Trans. Amer. Math. Soc. 167 (1972), $435-442$.

3. H. Behncke, A note on the Gel'fand-Nămark conjecture, Comm. Pure Appl. Math. 23 (1970), 189-200. MR 41 \#2404.

4. J. Dixmier, Les $C^{*}$-algèbres et leurs représentations, Cahiers Scientifiques, fasc. 29, Gauthier-Villars, Paris, 1964. MR 30 \#1404.

5. I. Kaplansky, The structure of certain operator algebras, Trans. Amer. Math. Soc. 70 (1951), 219-255. MR 13, 48.

6. C. E. Rickart, General theory of Banach algebras, University Series in Higher Math., Van Nostrand, Princeton, N. J., 1960. MR 22 \#5903.

DEPARTMENT OF MATHEMATICS, UNIVERSITY OF OREGON, EUGENE, OREGON 97403 